\title{
Integrating Reactive Cloud Applications in SERECA
}

\author{
Christof Fetzer \\ Technische Universität Dresden \\ Nthnitzer Str. 46 \\ Dresden, Germany \\ christof.fetzer@tu-dresden.de
}

\author{
Giovanni Mazzeo \\ University of Naples 'Parthenope' \\ Via Amm. Ferdinando Acton, 38 \\ Naples, Italy \\ giovanni.mazzeo@uniparthenope.it
}

\author{
John Oliver \\ jClarity \\ 32-28 Scrutton Street, EC2A 4RQ \\ London, United Kingdom \\ john@jclarity.com
}

\author{
Luigi Romano \\ University of Naples 'Parthenope' \\ Via Amm. Ferdinando Acton, 38 \\ Naples, Italy \\ luigi.romano@uniparthenope.it
}

\author{
Martijn Verburg \\ jClarity \\ 32-28 Scrutton Street, EC2A 4RQ \\ London, United Kingdom \\ martijn@jclarity.com
}

\begin{abstract}
A consolidated trend in designing cloud-based applications is to make use of a reactive microservice architecture, which allows to divide an application in several well-partitioned software units with specific responsibilities. Such an architecture perfectly fits in cloud environments, ensuring a number of advantages (i.e., high availability and scalability, ease of deployment and development). However, the new way of designing cloud applications introduces challenging security threats. Besides the difficulty in monitoring security of the overall distributed application, an important aspect of concern relates to the risk of break the chain of trust established among the different microservices belonging to the application. That is, a compromised single microservice may bring down the other related ones.

In this paper, we present the approach pursued in the context of SERECA ${ }^{1}$ project to secure microservice based applications. We leveraged the new extension of Intel's CPU, namely Software Guard eXtension (SGX), to enhance the security of applications using Eclipse Vert.x, the tool-kit for building reactive cloud applications. We developed an infrastructure composed by several SGX-enabled facilities (e.g. Database, Containers, Coordination Services) to support the process of integration between Intel SGX and micro-service applications. Our platform has been, then, validated through two use cases that made use of the developed secure facilities, i.e., a Critical Infrastructure (CI) monitoring application - having strong requirements in terms of data integrity - and an application for performance analysis of cloud-based services where the confidentiality of data is of main interest.
\end{abstract}

\section{KEYWORDS}

Cloud Security, Microservice, Intel SGX, vert.x

$\overline{{ }^{1} \text { https://www.serecaproject.eu }}$

Permission to make digital or hard copies of part or all of this work for personal or classroom use is granted without fee provided that copies are not made or distributed for profit or commercial advantage and that copies bear this notice and the full citation on the first page. Copyrights for third-party components of this work must be honored For all other uses, contact the owner/author(s).

ARES '17, Reggio Calabria, Italy

(C) 2017 Copyright held by the owner/author(s). 978-1-4503-5257-4/17/08 ..\$15.00 DOI: $10.1145 / 3098954.3105820$
ACM Reference format:

Christof Fetzer, Giovanni Mazzeo, John Oliver, Luigi Romano, and Martijn Verburg. 2017. Integrating Reactive Cloud Applications in SERECA. In Proceedings of ARES '17, Reggio Calabria, Italy, August 29-September 01, 2017, 8 pages.

DOI: $10.1145 / 3098954.3105820$

\section{INTRODUCTION}

Reactive microservice architecture is the modern way to architect cloud applications. This new typology of software design grew with the advent of cloud technology, as a result of an adaption process to the underlying distributed architecture. In contrast to monolithic applications, microservices are based on independent, autonomous, modular, self-contained units. The microservice architecture is quite often based on reactive principles, i.e., scalability, resiliency to failures, and message-driven. The new paradigm offers several benefits to both end users and developers. The firsts can take advantage of the high scalable, available, and flexible architecture. While, the latter can profit of the ease of development and deployment capabilities offered. The tremendous advantages coming from microservices does not come free of costs. In fact, the highly partitioned and agile architecture introduces new security issues. In particular, it is much more difficult to monitor security of the entire application spanned over several distributed systems, and, most important, since microservices are designed to completely trust each other, a single compromised microservice may bring down the entire application by breaking the chain of trust.

Our work aims at advancing the state-of-the-art of hardening mechanisms for developing secure cloud-based reactive applications having a microservice architecture. The approach pursued in the context of Secure Enclaves for REactive Cloud Applications (SERECA) project was to integrate the new security extension of Intel's CPU, namely Software Guard eXtension $(S G X)^{2}$, with Eclipse Vert. $x^{3}$. Intel SGX [5] is the recently released new technology for protecting applications through a hardware-enabled trusted execution. SGX is an instruction set extension, released in the Skylake CPUs, which allows the creation of Trusted Execution Environment (TEEs) inside the address space of an application. TEEs, known as enclaves in SGX jargon, provide strong protection of code and data

\footnotetext{
${ }^{2} \mathrm{https} / / / 01$. org/intel-softwareguard-extensions

${ }^{3}$ http://vertx.io/
} 
inside through encryption and integrity checks of their memory range, performed directly by the CPU.

Vert.x, instead, is a set of supporting tools for developers willing to implement microservice, event-driven, non-blocking applications. Core of vert.x is the distributed event bus, which allow microservices (known as verticles in vert.x jargon) to communicate with each other in a reactive and asynchronous way.

We present, in this paper, the developed software stack used to make microservice-based applications - running on top of vert.x - SGX enabled. So, besides describing at high-level the most important components of the SERECA platform that enable secure microservices (e.g. secure containers, secure storage systems, secure coordination service), we present a Critical Infrastructure (CI) use case chosen for validation purposes, and most important, how this leverages the secure facilities belonging to the SERECA platform.

The reminder of this work is organized as follows. Section 2 presents the most accepted approaches used nowadays to enhance cloud security. Such a section is followed by Section 3 that surveys the two main background technologies/tools, i.e., Intel SGX and Eclipse Vert.x. Then, in Section 4, we describe the techniques adopted to create an entire SGX-enabled software stack. Afterwards, in sections 5 and 6 , we show the use cases chosen to validate the secure developed platform. Finally, Section 7 concludes the paper.

\section{EXISTING APPROACHES TO CLOUD SECURITY}

A modern public cloud is home to a hardware and software stack consisting of thousands of devices, millions of lines of code, and arbitrary number of unknown tenants. The hardware is typically a mix of different models of different hardware generations, purchased in bulk more for their price than for their reliability. The software includes large frameworks, e.g., OpenStack, that are evolving rapidly and potentially harbor numerous exploitable bugs and configuration errors. The challenge for operators is to convince potential clients that it is safe to execute their applications and store their data in such a dangerous environment.

One approach taken by operators is to define a Trusted Computing Base (TCB) within the stack. Typically, the TCB will include most of the basic middleware, operating system (OS), and networking facilities of the data centre, as well as its hardware platform. Establishing the credibility of the TCB amounts to verifying the correctness and security of a large and complex hardware and software system. Aside from the high cost of doing so continously as the hardware and software evolve, the goal of a truly "trustworthy" TCB has proven elusive $[7,8]$.

Even if it were possible to remove all bugs from the TCB, this alone will not ensure the security of cloud applications, since a malicious system administrator employed by the cloud operator could exploit their privileges to access customer data while the data are, for example, unencrypted in main memory or CPU registers.

An approach focused specifically on securing application data from access by both external and internal malicious agents is based on the use of homomorphic encryption, which is a technique intended to allow computations to be carried out directly on encrypted data $[3,6,10]$. The promise of this technique is that the unencrypted form of the data would never need to be present within the data centre, and so never exposed to attack. The technique also extends to the computations themselves, so that they too could be encrypted and thereby protected from exposure. Unfortunately, the goal of homomorphic encryption is proven as elusive as that of a trustworthy TCB $[12,13]$. The computations for which the technique is known to work are currently quite limited, making their use in most normal applications infeasible. Moreover, homomorphic encryption is notoriously slow, making its use in reactive applications untenable.

A third approach being investigated for the cloud is the use of specialised security co-processors. Such processors consider the chip area as a trust boundary, treating everything outside as subject to attacks and potentially compromised. As in the case of homomorphic encryption, the instructions and data are stored encrypted in the memory. However, once read by the processor, they are decrypted and the instructions carried out on plain-text code and data. Because everything outside the chip can be tampered with, the chip never outputs plain-text, encrypting the data before writing to the bus. While secure processors provide good security guarantees, the notion of investing in specialised hardware is counter to the general principle of data centre "scale out", which advocates the use of large numbers of low-cost commodity components. Another disadvantage is that the use of such processors is not transparent to developers, because they need to specifically tailor their applications to leverage features provided by the hardware $[9,11]$.

\section{BACKGROUND}

In this section a brief description of main concepts behind Intel SGX and Eclipse Vert.x is reported. We give a short introduction on main benefits coming from both of them.

\subsection{Intel SGX}

Intel SGX [5] can be conceived as a "Reverse Sandbox", which aims to protect the integrity and confidentiality of code and data by creating a reserved memory space known as enclave able to protect an application from the external environment (including the OS or the Hypervisor). The enclave contains the sensitive data and the code that uses it. By doing so, SGX enables users to enforce the security of their code and data without having to trust the cloud provider. SGX is a new instruction set extension, which has been released in the Skylake processor generation. Enclaves are protected by the CPU package itself from a number of critical security threats: enclave memory is encrypted by the CPU limiting trust to the CPU package itself.

\subsection{Eclipse Vert.x}

Vert.x is a tool-kit for building reactive applications on the Java Virtual Machine. In the context of Vert.x, micro services are called verticles, and are supposed to comprise a scarce, well-defined part of application logic. Vert.x is polyglot, it can be implemented in various programming languages and interact across programming languages. Verticles communicate via an event bus that connects verticles even across machine boundaries were they can subscribe an address in order to receive callbacks once a message arrives. All verticles are scheduled via the event loop thread of Vert.x (one per 
physical CPU core), which delivers events to verticles. Supporting the idea of reactive applications, a verticle should never block this thread and implementing I/O operations in an asynchronous fashion.

\section{THE SERECA PLATFORM}

In this section a number of features belonging to the SERECA platform are overviewed. More precisely, the following are going to be described: 1) The extension developed to bring secure hardening mechanisms into vert.x; 2) The chosen methodology to run vert.x verticles into SGX enclaves and let them communicate with the event bus; 3) The SGX-enabled containers useful for secure microservices deployment; 4) The secure coordination service harden with SGX; 5) The secure storage services that store data encrypted into SGX enclaves; 5) The architecture of the cloud platform having CPUs with the Intel SGX extension.

\subsection{Secure Vert.x}

A core component of vert.x is the Event Bus, which allows the asynchronous communication between verticles. Due to its role, the security of the event bus is of paramount importance. The SERECA platform, therefore, provides a Secure vert.x, which includes a $S e-$ cured Event Bus and an Encryption Service. The Secured EventBus enables message- and transport- based encryption mechanisms into the vert.x framework.

The new secured version of vert.x enforces the Transport-based Encryption for the event event bus, for all members taking part in the communication through Transport Layer Security (TLS). Additionally Message based Encryption is provided through symmetric $A E S$ with 256 bits keys to ensure confidentiality and a HMAC mechanism (based on SHA256) to ensure integrity. Based on the address of the message exchanged, the payload of a message is encrypted when an event is sent using a specific key. Therefore, only receivers knowing the encryption key (AES) and integrity key (HMAC) can decrypt the message.

\subsection{SGX-Vert.x JNI Bridge}

Eclipse Vert.x is written in Java, while SGX enclaves are written in $\mathrm{C} / \mathrm{C}++$. Hence, running vert.x verticles into SGX enclaves would result in porting a Java Virtual Machine (JVM) into them. The integration of a full-edged JVM into an enclave would be possible in theory, however, this introduces a lot of complexity to the enclave and also causes a drastic increase of the TCB. This would lead to a higher probability of exploitable security vulnerabilities and increase the attack surface.

Hence, our approach was to leverage the Java Native Interface (JNI) to create a sort of bridge, namely Vert.x Vault, between vert.x (Java) and SGX $(\mathrm{C} / \mathrm{C}++)$. In order to complete the secure data processing new Intel $S G X$ protected native components need to be built and then accessed via the JNI bridge. The workflow for using this new bridge is as follows:

(1) Vert.x event bus messages arrive at the Java side of the JNI bridge (registered to it on the enclave's behalf), the transport layer encryption stops here.
(2) The Java side of the bridge passes the message (still protected by message level encryption) through to the native side of the bridge.

(3) The native side of the bridge passes the message to a native component running inside the enclave for decryption and processing.

The following diagram shows the how the JNI bridge fits into the architecture.

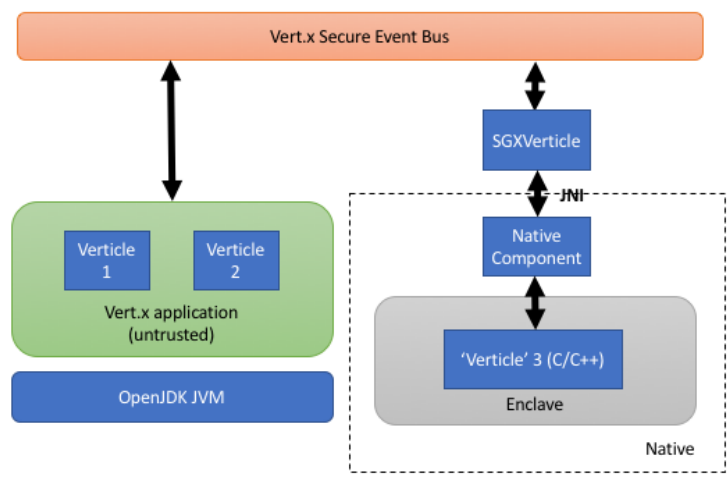

Figure 1: JNI Bridge between Eclipse Vert.x and Intel SGX

\subsection{Secure Coordination Service}

Coordination is an essential component in many distributed systems. In SERECA, ZooKeeper (ZK) has been chosen as the coordination service. An extension of ZooKeeper called SecureKeeper[2] was developed. The secure ZooKeeper extension makes use of secure enclaves in order to protect the payload of all znodes. To achieve this secure enclaves must be put into the communication channel between ZooKeeper clients and replicas. The resulting architecture of secure ZooKeeper is shown in Figure 2.

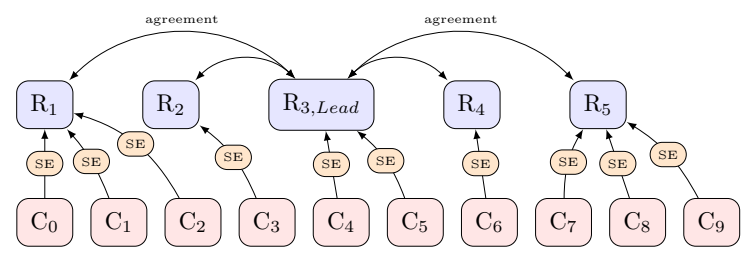

Figure 2: Architecture of Secure ZooKeeper

The basic idea is to have the enclave process each message from and to the ZooKeeper replicas and protect the confidentiality of all sensitive data contained in the communication. For this purpose, the enclave will process each request message in the same way, responses are handled in reverse order:

(1) Decrypt whole message (Transport Encryption)

(2) Deserialize the message

(3) Process message to protect confidential daya (Encrypt Payload) 
(4) Serialize the message

The idea is to add a simple transport encryption for all messages on the client side. Before the encrypted messages reach the replica they are forwarded to the secure enclave which decrypts and analyses the messages. The secure enclave understands (i.e. deserialize) the meaning of the message to some extend, so it can isolate the payload of the znode and encrypt only the payload field of the message, while keeping the plaintext of the other fields.

Afterwards, the message is forwarded to the ZooKeeper replicas and processed normally. This allows to easily protect the confidentiality - and if a HMAC is added, also the integrity - of all data stored inside the znode's payload. In addition, this approach is completely transparent to the ZooKeeper replicas, since no direct processing of the znode's payload is done by them.

\subsection{Secure Storage Services running in Secure Containers}

Another aspect that deserves particular attention relates to the storage systems. For example, the CI use case (deepened later) imposes as security requirement that data is kept stored in a secure manner. In SERECA two storage systems have been hardened, i.e., MongoDB and MySQL. The two were chosen based on the functional requirements imposed by the use case, i.e., having separated storages for time-recent and historical data. The platform, therefore, embeds a two extended version of MongoDB and MySQL which make use of SGX enclaves. These run into SGX-enabled containers developed in the context of SERECA.

In fact, in SERECA we provide enclave-enabled applications in the cloud through a popular deployment mechanism, that is: Docker containers. The platform includes a secure runtime to allow configuration and execution of enclave applications in untrusted environments. The idea is to execute critical processing inside containers (3) that will be more easily deployed on top of SGX. The project goes under the name of SCONE (Secure Linux Containers with Intel $S G X)$ and is described by Arnautov et al. [1] in a recent paper. Hence, both MongoDB and $M y S Q L$, are hardened by running them into SCONE secure containers.

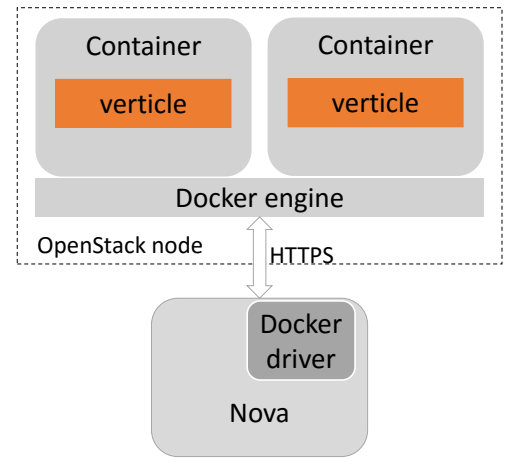

Figure 3: Secure Deployment with Docker Containers

\subsection{SGX Bare-Metal Cloud Platform}

A standard cloud stack cannot be used in conjunction with Intel SGX. In fact, there currently are no hypervisors with SGX support available. To overcome such a limitation and so find a way of testing the hardened use case application, we opted for a Bare-Metal Cloud, on which the SERECA platform will reside. This is a Metal as a Service (MaaS) platform, and allows its users to dynamically allocate physical machines, as opposed to virtual machines. Since the different facilities need access to the SGX features of underlying hardware, it is advantageous to have access to the physical machines since this allows us to circumvent the problem that there currently are no hypervisors with SGX support available. Using the MaaS platform, however, there is no need for a hypervisor, and we can install our own operating system, i.e., Linux with SGX support, allowing us to get full access to the SGX features of the underlying hardware.

The MaaS platform has a web based front end, where you can configure your host and deploy services, repeatedly and reliably. Among many things, it supports remote setup, open-source IP address manager, integration with devops automation tools and has an integrated SSH forwarding mechanism.

The MaaS platform consists of one cluster of four SGX-enabled hosts, which are managed by a server controlling the SGX servers via the IPMI management interface over a separate network. The four SGX enabled nodes have the following hardware specification: Intel Xeon E3-1230v5 QuadCore with Hyperthreading (S-SPEC: SR2LE); Supermicro X11SSZ-F Mainboard; 8 GB DDR4 RAM (upgrade to 32 GB will be performed in the following months); 256 GB PCIe NVMe SSD storage.

\section{USE CASE \# 1: SECURE CLOUD-BASED MONITORING SYSTEM FOR A CRITICAL INFRASTRUCTURE}

We chose a civil Water Supply Network Monitoring (WSNM) infrastructure as a use case to validate and demonstrate the effectiveness of our work. We developed an application, namely RiskBuster, that makes use of the facilities previously described in section 4 . The WSNM is a valid example of a critical infrastructure having strong security requirements. In fact, the use case - under the administration of a public authority namely Ente per lo Sviluppo dell'Irrigazione e la Trasformazione Fondiaria in Puglia, Lucania ed Irpinia (EIPLI) provides water to a large part of Southern Italy population. EIPLI is in charge of the distribution of water - around 600 million cubic meters per year - for different uses (e.g. industrial, energetic, irrigation, drinking) to the nearest population. Besides the management of the water pipelines, the organization is also responsible of 8 dams with different storage capacities up to $550 \mathrm{Mmc}$.

The administrators of EIPLI - following the current trend of CIs cloudification - aims at moving their real-time monitoring application in a cloud environment. This is attractive as they can discern from the burden of IT systems managements costs and easily interconnect all the assets possessed, which are geographically distant between each other. Furthermore, except for the aspects of security, the choice of adopting cloud technologies is further strengthened by the non-functional requirements imposed and briefly defined in 5.1 . 


\subsection{Imposed Requirements}

According to Cerullo et al. [4] work, the EIPLI's infrastructure imposes the following requirements.

5.1.1 Functional. Main monitoring functionalities needed by the WSNM application consist in: 1) Data acquisition from sensors, meters and field devices, such as photo, pressure, temperature and flow sensors needed to monitor fundamental parameters for the water quality and the infrastructure integrity; 2) Data collection, storage and retrieval for subsequent analysis such as statistical trends, regulations, future load planning or billing; 3) Alarm detection and management to find any suspicious activity or abnormal situation on the infrastructure by correlating events of different nature; 4) Monitoring in real-time the status of the WSNM infrastructure to react to possible alerts or alarms, and extracting historical trends.

5.1.2 Non-Functional. The WSNM is unarguably exposed to risks that are related to natural phenomena, disasters, and criminal/terrorist activity. For this reason, the non-functional requirements imposed, especially those related to the security and dependability, are particularly stringent. These are the cause of the skepticism to promote a serious migration of CI applications to the cloud. The monitoring application should guarantee: 1) Data security since critical decisions are usually taken using the measurement data provided by the monitoring system, meaning that data confidentiality and, especially, data integrity are of primary importance; 2) Service availability and responsiveness to ensure a continuous service provision, and a quick reaction of operators critical conditions; 3) Scalability in order to tolerate load peaks of the processing throughput. This capability must be maintained also for an increased number of feeding components (i.e. more sensors or event analysers). 4) Interoperability to support an easy integration of technologies of different nature and also allow the usage of software modules written in different programming languages.

\subsection{Microservice-based Design of the WSNM Monitoring Application}

An important step required for the use case integration with the facilities of the SERECA platform (described in 4) was the migration from a monolithic to a microservice software architecture, making the RiskBuster application easily deployable in cloud environments. Hence, based on the functional requirements, we identified a number of units to be associated with as many microservices (or verticles). Figure 4 reports them. On the dam-side, a data collector verticle interfaces, through a ModBus protocol, with a data logger equipment responsible for providing all the sensor data. Then, the acquired measurements are sent to the subscribed cloud-verticles through the vert.x event bus.

On the cloud-side, four verticles receive data through the event bus and, based on their duties, take a specific action on it. These are:

Cache Archiver Verticle (CAV) - It is responsible for the storage of time-recent data into an in-memory system. Such a data is needed, e.g., by the alarm manager in charge of real-time analysis activities.

Alarm Manager Verticle (AMV) - The alarm manager is in charge of signaling dangerous situations occurring on the dam infrastructure by enforcing a Complex Event Processing (CEP), i.e., correlating different data sources to find events or patterns suggesting more

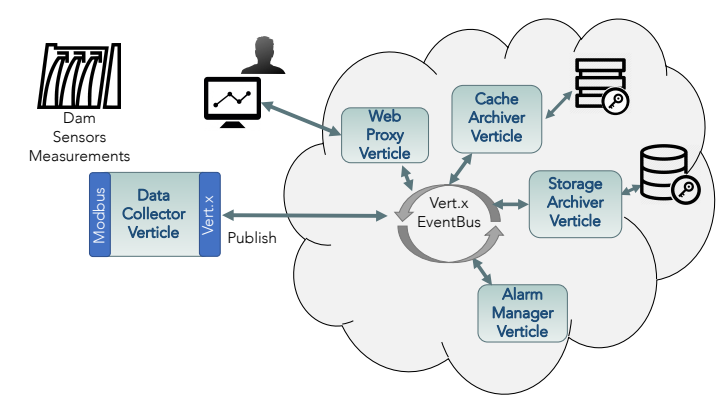

Figure 4: Microservices belonging to the WSNM application

complicated circumstances. To do that, the AMV needs to receive live data from the collector verticle and also temporarily store data in memory. For this reason, it communicates with the CAV through point-to-point messages exchanged through the secure event bus.

Storage Archiver Verticle (SAV) - Beside saving data for CEP processing purposes, it is also important to store measurements for historical trend evaluations into a persistent storage system.

Web Proxy Verticle (WPV) - The final user operator has access to the monitoring application through a web-based dashboard, which reports real-time measurements, historical trends, and alarms notifications. Such a dashboard communicates with the back-end cloud application through the WPV. The WPV-based on user requestscan ask to any verticle for data to send to the web browser.

\subsection{Integration of SERECA Facilities}

Once designed RiskBuster as a microservice application, we integrated the secured facilities of the SERECA platform. Figure 5 reports the secured architecture of the WSNM application where all the hardening mechanisms are reported. In the rest of this section, the different processes of facilities integration will be surveyed.

5.3.1 Secure Vert.x. A first integration activity consisted in migrating from the standard vert.x to the secured one. The different verticles that handle sensitive data, now communicate through encrypted messages. In the new RiskBuster architecture, sensitive sensor measurements are encrypted at each level of communication: between the on-field data collector verticle and the vert.x on-cloud gateway, among cloud application verticles (i.e., Mongo Archiver, MySQL Archiver, Web Proxy, and AMS), and between the Web Proxy verticle and the web dashboard GUI/HMI. The application now leverages the new Secure Event Bus that enforces messageand transport- based encryption on the verticles communication. Each verticle involved in the application instantiates a SecuredEventBus object by defining the address and the key to be used, and also an Encryption Service responsible of encryption/decryption of messages.

Four keys have been used in RiskBuster to harden the communication between different type of verticles: one for Data PublisherGateway communication, one for the Gateway and the different subscribed verticles, one for the AMS verticle and the Web-Proxy, and finally one to secure the Web-Proxy-Dashboard communication. Any time a verticle wants to send something on the Secure EventBus, it encrypts the message through the Encryption Service 
providing the id of the key used. Clearly, on the other side, the receiver verticle(s) will decrypt the encrypted message with the same key.

Furthermore, RiskBuster makes use of a Secured SockJS Bridge. This includes new revised APIs that enforces encryption/decryption of messages also at browser level. In this way, sensor measurements sent from the Web-Proxy to the web-based Dashboard will be secured using a key shared between the application and the browser.

5.3.2 SGX-Vert.x JNI Bridge. Sensitive sections of RiskBuster must run into the enclave in order to leverage the strong SGX hardening mechanisms. The set of JNI APIs interacting with the SGX enclave have been therefore leveraged to exploit the SGX enclaves. In particular, we used those functions that allow to register/unregister to/from a specific event bus topic, send point-to-point messages, or publish messages to subscribed verticles. Hence, we define the notion of a secure verticle that essentially represents a verticle whose application logic is implemented inside an enclave. In the WSNM application, three secure verticles replace the unsecured ones. Here we report them:

Secure Alarm Manager (SAM) - This secure verticle enforces complex event processing on sensors data into a SGX enclave. It is subscribed to specific topics (or sensors) of interest through the ocall_register() function provided by the JNI bridge. During the execution, the secure alarm manager continuously receives updates directly into the enclave and process them to find patterns that suggest alarm conditions.

When needed, the SAM asks to the Secure MongoDB Archiver (i.e., the CAV verticle) time-recent data for additional evaluations. This is realized through a point-to-point message exchange, enabled by the JNI bridge. The communication occurs through the secure event bus and, most important, terminates - on both sides - into SGX enclaves.

Alarms must be clearly notified to operators through the web dashboard. Hence, the SAM interacts with the Secure WebProxy in order to let it forward, in a secure manner, potential notifications. Beside being encrypted, these are sealed into the enclave with the purpose of avoiding integrity attacks.

Secure MongoDB Archiver (SMDB) - The SMDB currently collects measurements into the enclave similarly to the SAM by registering from within the enclave - through the JNI bridge - to the secure event bus.

What is still missing is the final storage into the secure containers. The idea is that the when updates are received, the SMDB establishes an enclave-terminated TLS connection with the Secure Container (SCONE), which wraps the SGX-enabled MongoDB storage system The rationale behind the enclave-terminated TLS communication is to keep the TCB restricted to the SGX enclaves. That is, the OS is considered a source of threat and cannot handle the TLS connection. The data is always transmitted encrypted and it is decrypted only within an enclave ensuring an extremely high level of security. Hence, using the established secured connection, the SMDB will call the dedicated APIs used to drive MongoDB operations, e.g., store/delete/query measurements of interest. These are indirectly performed by more than one verticle, i.e, the Secure Alarm Manager (SAM) and the Secure Web Proxy.

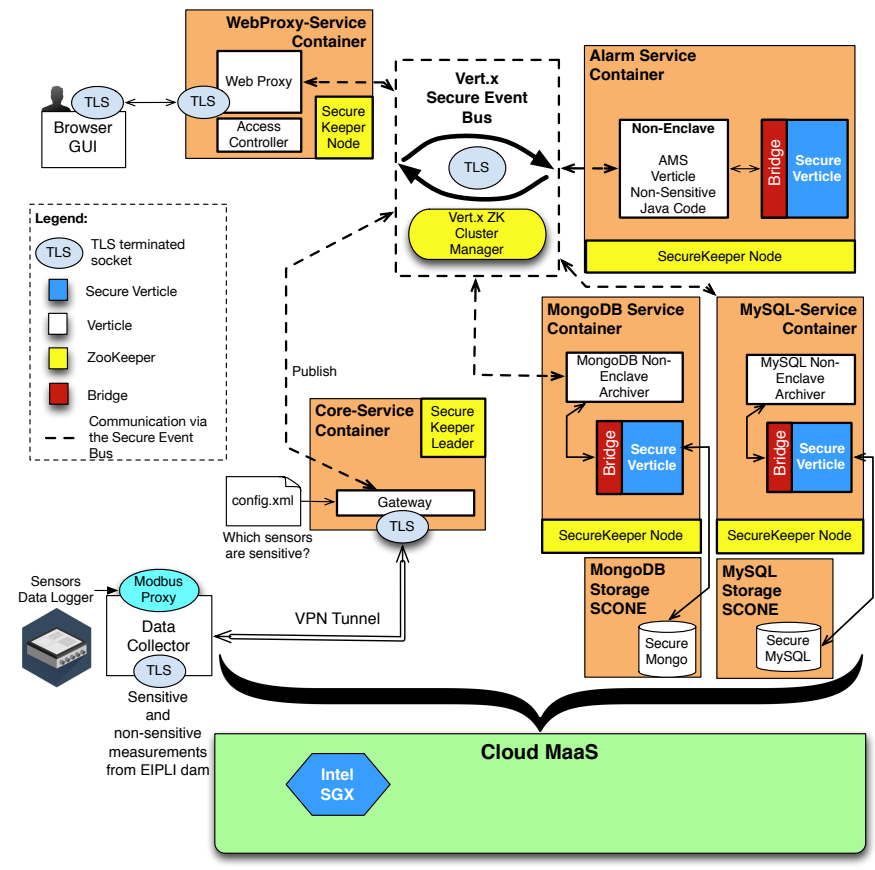

Figure 5: Architecture of WSNM application with Integrated Facilities

Secure MySQL Archiver (SMSQL) - The SMSQL will act similarly to the SMDB. That is, it will receive real-time data and send it to a secure MySQL storage using the enclave-terminated TLS connections. The SMSQL establishes a point-to-point communication with the secure mongo archiver by asking with an ocall_send() a bulk of data to store into the historical database.

5.3.3 Secure Coordination Service. The integration of SecureKeeper has been almost transparent for RiskBuster. In fact, the WSNM application interfaces with the coordination service through specific vert.x APIs that, in turn, manage the verticle execution on top of the cluster manager. The initial version of the pilot made use of those vert.x functions dedicated to the interaction with the Hazelcast cluster manager. The secured pilot application - due to the SecureKeeper migration - required a new set of APIs to be included in order to allow the communication with the updated coordination service. Hence, from the pilot application point of view, a new set of vert.x Java APIs have been included. These allow to start-up the coordination service and to discover new members (i.e. verticles) to be added to the cluster.

5.3.4 Secure Storage Services running in Secure Containers. A dockerized version of the pilot application has been released in order to allow the ease deploy into the MaaS cloud platform and integrate the secure storage services, running in SCONE. A DockerCompose $^{4}$ extension that allows to define multi-container Docker applications. The core idea of Docker-Compose is to define into a Compose .yml configuration file the needed services of an application (e.g. MySQL, MongoDB) and then execute each of them into

${ }^{4}$ https://docs.docker.com/compose/ 
single monolithic containers.

Hence, using docker-compose we defined a distributed application in which each verticle, and each service (i.e., MySQL and MongoDB) run into single Docker containers. Our application is therefore composed of several containers where two of them (the storage services ones) are SGX-enabled.

\section{USE CASE \#2: SECURE APPLICATION PERFORMANCE ANALYSIS SERVICE}

\subsection{Business Use Case}

Illuminate is an Application Performance Management (APM) platform driven by Machine Learning (ML). It is delivered to jClarity's (JC) customers via Software as a Service (SaaS) hosted on public cloud infrastructure. Customers that use Illuminate need to have a subset of the overall data transmitted, processed and stored in a secure manner. This in effect means being protected from cloud provider employees and/or external attackers with root access to the underlying cloud platform. In particular Diagnosis reports and Daemon / Host information need to be secured in this manner. Illuminate Daemons run on the client's hosts and communicate via a secure TLS WebSocket connection to the Illuminate Reporting Agent. It is important to note due to restrictions on running on our client's hosts, the Daemons are not part of the Vert.x cluster, and are not able to run a SecureKeeper node. That is, only when data hits the Illuminate Reporting Agent do we have access to the vert.x Secure EventBus and the rest of the SERECA platform.

\subsection{Initial Integration of illuminate with SERECA Facilities}

The earliest redesign of the illuminate application was focused on preparing it for a microservices design based on the concept that certain data flows were sensitive and therefore had to be processed securely by the SERECA Cloud Platform. Illuminate therefore was upgraded to the latest secure version of Vert.x, and also split its data processing services and data abstraction services so that secure data flows were isolated.

Illuminate was then extended with the Vert.x Secure Event Bus APIs developed in the context of SERECA by Red Hat. Through the Secure Eventbus, most security-critical micro-services of illuminate now support encryption and can exchange sensitive data in a secure manner. That is, messages, before being sent, are securely encrypted. The encryption is enforced at both application (i.e., Message-based encryption) and transport (TLS) layers. Only the involved receiver inside the enclave, knowing the encryption key (AES) can decrypt the message. This approach does rely on an assumption that the key stored on the Daemon on the client's host is deemed to be secure (possibly even "SGX secured" if our customers adopt this technology).

Furthermore, the Vert.x secure APIs enable illuminate to handle security when a web client accesses the application. This is done through a Secured SockJS Handler which establishes secure communication between the on-cloud application core and the client web browser dashboard.. This approach does rely on the assumption that the key stored on the browser on the end user's machine is deemed to be secure. Because the TLS connection terminates

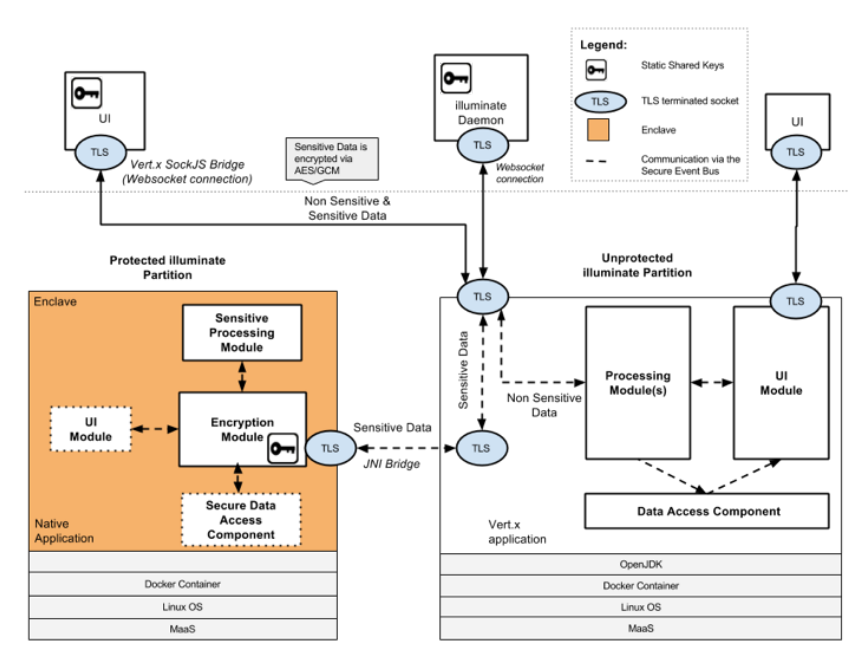

Figure 6: Architecture of APAS application

in a vertx web server endpoint (on non SGX enabled Java) extra message level encryption is also used to guarantee confidentiality. Additional integration work includes the Docker-based deployment on the Metal-as-a-Service(MaaS) cloud infrastructure.

Figure shows the final architecture of illuminate currently running on top of the MaaS cloud infrastructure. The application receives data from Daemons running on customer hosts, processes sensitive data in a secure manner (encryption and anonymization, backed by SGX) and allows users to securely view that data. The communication between services is facilitated by the Vert.x secure event bus that enforces a transport- and application- level encryption.

jClarity has re-architected Illuminate in order to reduce the TCB and to ensure that there is end-to-end encryption of sensitive data from the Daemon into the SERECA platform. Once in that platform, SERECA features such as the Vert.x secure eventbus, JNI Bridge et al are used. The architecture is split up broadly as follows:

SGX Secured Partition - A native $(\mathrm{C}++)$ component running on the native side of the JNI Bridge that interacts directly with the SGX secure enclave. Decryption and processing of secure data happens within this partition. Non SGX Partition - A vert.x instance running on top of a regular OpenJDK / Oracle JDK JVM (outside any SGX secure enclaves) for non sensitive data processing and storage. SSL based security and authorisation are still used, but are not protected specifically by any SGX enhanced capabilities.

Illuminate Daemons run on the client'fis hosts and communicate via a secure TLS WebSocket connection to the Illuminate Reporting Agent. Sensitive information is encrypted by the shared key with the enclave. The enclave will decrypt the this data using it's Daemon key, perform some processing and then re-encrypt using its UI key for the exchange of data to the browser (end user).

\subsection{JNI Bridge Usage}

As described in the Facilities provided section, a new JNI bridge is used to transmit encrypted messages to a SGX enabled native 


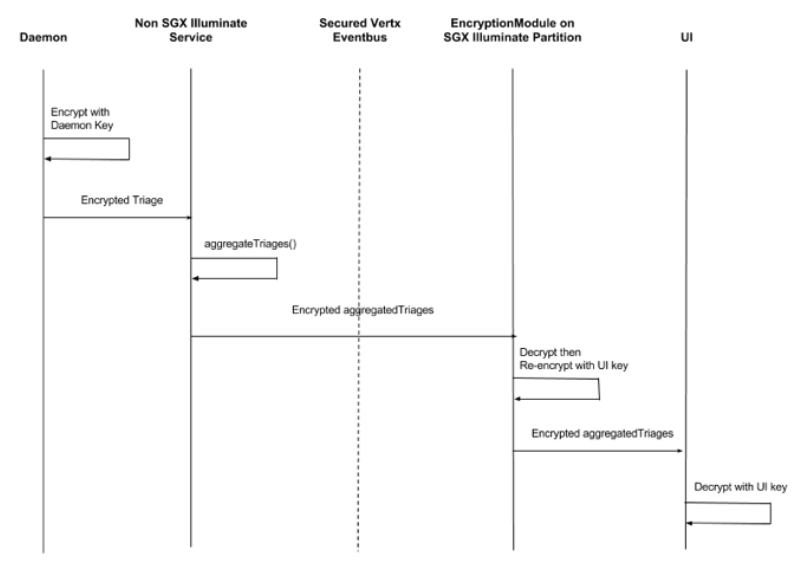

Figure 7: Illuminate Daemon and Illuminate Service encryption using Shared Keys.

component for decryption and processing. An example flow for the triage message use case from the illuminate application follows:

(1) An encrypted message containing sensitive triage information arrives at the Java side of the JNI bridge (registered to it on the enclave'fis behalf), the transport layer encryption stops here.

(2) The Java side of the bridge passes the encrypted triage message through to the native side of the bridge.

(3) The native side of the bridge passes the encrypted triage message to a native component running inside the enclave for decryption.

(4) The native component processes the message for consumption by the UI and re-encrypts it into a new encrypted message (using a new separate key).

(5) The native component passes the UI encrypted message back through the bridge to the Java side.

\subsection{Lessons Learned}

We learned several lessons from building an industrial application on top of SERECA functionality. To ensure the smallest possible TCB of the native code running in an SGX enclave, there has to be significant application logic re-architecture work. There are also further challenges, of which the top three are:

(1) Re-designing the application data processing to have a TCB that fits inside the restricted SGX enclave memory size.

(2) Finding SGX enabled versions of modern $\mathrm{C}++$ language constructs and libraries errors in an enclave.

(3) Debugging native code errors in an enclave (restricted tooling and debug support)

Although there are significant challenges for a traditional Java application developer team, we expect that as SGX matures that these challenges will be mitigated. In the shorter term, increases in the SGX's memory limit and a growing SGX enabled native library ecosystem, will make it easier for traditional Java application teams. A long term goal for SGX would still be to have a full port of a Java runtime such as OpenJDK's HotSpot.

\section{CONCLUSION}

This paper presented an entire infrastructure, i.e., the SERECA platform, able to run microservice based applications in untrusted cloud environments. We showed, then, how the most relevant facilities of this platform can be leveraged by two relevant use cases, i.e., a Water Supply Network Monitoring system that unarguably belongs to Critical Infrastructures and a performance analysis application for cloud-based services.

\section{ACKNOWLEDGMENTS}

This project has received funding from the European Union's Horizon 2020 Research and Innovation Programme under Grant Agreement No.: 645011

\section{REFERENCES}

[1] Sergei Arnautov, Bohdan Trach, Franz Gregor, Thomas Knauth, Andre Martin, Christian Priebe, Joshua Lind, Divya Muthukumaran, Dan O'Keeffe, Mark L. Stillwell, David Goltzsche, Dave Eyers, Rüdiger Kapitza, Peter Pietzuch, and Christof Fetzer. 2016. SCONE: Secure Linux Containers with Intel SGX. In 12th USENIX Symposium on Operating Systems Design and Implementation (OSDI 16). USENIX Association, GA, 689-703. https://www.usenix.org/conference/osdi16/ technical-sessions/presentation/arnautov

[2] Stefan Brenner, Colin Wulf, David Goltzsche, Nico Weichbrodt, Matthias Lorenz, Christof Fetzer, Peter R. Pietzuch, and Rüdiger Kapitza. 2016. SecureKeeper: Confidential ZooKeeper using Intel SGX. In Middleware. ACM, 14.

[3] Craig Gentry. 2010. Computing Arbitrary Functions of Encrypted Data. Commun. ACM 53, 3 (March 2010), 97-105. DOI : http://dx.doi.org/10.1145/1666420.1666444

[4] Gaetano Papale Luigi Sgaglione Rosario Cristaldi Gianfranco Cerullo, Giovanni Mazzeo. 2016. A Secure Cloud-Based SCADA Application: The Use Case of a Water Supply Network.

[5] Intel Corp. 2014. Software Guard Extensions Programming Reference, Ref. 329298-002US. https://software.intel.com/sites/default/files/managed/48/ 88/329298-002.pdf. (Oct. 2014). https://software.intel.com/sites/default/files/ managed/48/88/329298-002.pdf

[6] Seny Kamara and Mariana Raykova. 2013. Parallel Homomorphic Encryption. In Workshop on Applied Homomorphic Encryption (WAHC '13).

[7] Jonathan M. McCune, Bryan Parno, Adrian Perrig, Michael K. Reiter, and Arvind Seshadri. 2008. How low can you go?: recommendations for hardware-supported minimal TCB code execution. In ASPLOS, Susan J. Eggers and James R. Larus (Eds.). ACM, 14-25.

[8] Ahmad-Reza Sadeghi, Marcel Selhorst, Christian Stble, Christian Wachsmann, and Marcel Winandy. 2006. TCG inside?: a note on TPM specification compliance. In STC, Ari Juels, Gene Tsudik, Shouhuai Xu, and Moti Yung (Eds.). ACM, 47-56.

[9] G. Edward Suh, Dwaine Clarke, Blaise Gassend, Marten van Dijk, and Srinivas Devadas. 2003. AEGIS: Architecture for Tamper-evident and Tamperresistant Processing. In Proceedings of the 17th Annual International Conference on Supercomputing (ICS '03). ACM, New York, NY, USA, 160-171. DOI : http://dx.doi.org/10.1145/782814.782838

[10] M. Tebaa, S. El Hajji, and A. El Ghazi. 2012. Homomorphic encryption method applied to Cloud Computing. In Network Security and Systems (FNS2). 86-89.

[11] David Lie Chandramohan Thekkath, Mark Mitchell, Patrick Lincoln, Dan Boneh, John Mitchell, and Mark Horowitz. 2000. Architectural Support for Copy and Tamper Resistant Software. In Proceedings of the Ninth International Conference on Architectural Support for Programming Languages and Operating Systems (ASPLOS IX). ACM, New York, NY, USA, 168-177. DOI : http://dx.doi.org/10.1145/ 378993.379237

[12] Marten Van Dijk, Craig Gentry, Shai Halevi, and Vinod Vaikuntanathan. 2010. Fully homomorphic encryption over the integers. In Advances in CryptologyEUROCRYPT 2010. Springer, 24-43.

[13] Zhenfei Zhang, Thomas Plantard, and Willy Susilo. 2011. Reaction Attack on Outsourced Computing with Fully Homomorphic Encryption Schemes. In ICISC, Howon Kim (Ed.). Springer, 419-436. 